\title{
Chatuphalatika, a Thai herbal formula, ameliorate obesity and dyslipidemia in high-fat diet fed mice
}

Salin Mingmalairak

Chiang Mai University

Mayuree H Tantisira

Burapha University

Prasoborn Rinthong ( $\nabla$ prasoborn.r@msu.ac.th )

Mahasarakham University

Research

Keywords: Chatuphalatika aqueous extract, Acute toxicity, High-fat diet, Dyslipidemia

Posted Date: May 21st, 2020

DOI: https://doi.org/10.21203/rs.3.rs-29053/v1

License: (1) This work is licensed under a Creative Commons Attribution 4.0 International License.

Read Full License 


\section{Abstract \\ Background}

Chatuphalatika is a Thai traditional health tonic composing of four different herbs namely Terminalia bellerica Linn., T. chebula Retz., T. arjuna Roxb. and Phyllanthus emblica Linn. The fact that phytoconstituents of Terminalia species have been reported to ameliorate obesity and symptoms of metabolic syndrome prompt us to investigate acute toxicity as well as a lipid lowering activity of orally given chatuphalatika aqueous extract (CPT) in animal models.

\section{Methods}

CPT was prepared by decoction method and the phytochemical contents were quantified by HPLC analysis. The acute oral toxicity study of CPT was performed in Wistar rats following the protocol of the Organisation for Economic Co-operation and Development (OECD) guidelines No. 420. The assessment of lipid-lowering effect of CPT was carried out in high-fat diet (HFD) fed C57BL/ 6 mice model.

\section{Results}

Gallic acid was the highest content (137.10 $\pm 5.42 \mathrm{mg} / \mathrm{g}$ ) found in CPT followed by chebulinic acid $(73.60 \pm 2.35 \mathrm{mg} / \mathrm{g})$, chebulagic acid $(62.60 \pm 4.17 \mathrm{mg} / \mathrm{g})$ and ellagic acid $(5.2 \pm 0.40 \mathrm{mg} / \mathrm{g})$. No lethality and no signs of toxicity were observed in either male or female rats orally treated with CPT at a single dose of 2,000 and $5,000 \mathrm{mg} / \mathrm{kg}$. Daily oral administration of 250,500 or $1,000 \mathrm{mg} / \mathrm{kg}$ of CPT for 6 weeks to the HFD-induced dyslipidemia mice markedly reduced low density lipoprotein cholesterol (LDL-C) levels and increased high density lipoprotein cholesterol (HDL-C) levels, whereas the significant reduction of total cholesterol (TC) levels was observed only in the high doses of 500 and 1,000 mg/kg of CPT. Moreover, dyslipidemia mice received $1,000 \mathrm{mg} / \mathrm{kg}$ CPT exclusively showed the decrease in body weight gain.

\section{Conclusions}

The CPT was safe and effective for relieving dyslipidemia and reducing weight gain in animal model supporting further development as functional food for dyslipidemia or overweight population.

\section{Background}

Coronary vascular disease (CVD) and its complications are the leading cause of mortality in the world [1]. Obesity and dyslipidemia have been associated with an increased risk of CVD and adverse cardiovascular events [2]. The prevalence of overweight and obesity has dramatically increased worldwide since 1980 and these metabolic disorder are one of the major public health problems of this 
century $[3,4]$. Dyslipidemia is characterized by elevated the blood total cholesterol (TC), low density lipoprotein cholesterol (LDL-C) and very low density lipoprotein cholesterol (VLDL-C) levels and reduced high density lipoprotein cholesterol (HDL-C) levels. Several methods are presently practiced to control the cholesterol levels. 3-hydroxy-3-methyl-glutaryl-coenzyme A (HMG-CoA) reductase inhibitors or statins have been proven to be the most efficient therapy to balance the blood cholesterol levels, however, on expenses of its adverse effects.

Basic theory of Thai traditional medical concerns the perfect balance and harmonize of human body elements. The belief of health care and medical treatment of Thai people is also approached from a holistic perspective [5]. Therefore, herbal formulation rather than single plant extract or isolated compound has been widely use to manage the illness [(6)]. Chatuphalatika is a Thai health tonic formulation traditionally used as the laxative, colon cleansing and detoxifying agents [(7)]. Name of the formulation means "four" (chatu) "fruits" (phala) as it is consisted of equal parts of the Terminalia chebula Retz., T. bellerica Linn., T. arjuna Roxb. (Combretaceae) and Phyllanthus emblica Linn. (Euphorbiaceae).

At present, published scientific data of chatuphalatika are scare. Our previous study reported the antioxidant, anti-inflammatory and antihyperuricemia effects of chatuphalatika extract [8]. However, the individual fruit constituent of chatuphalatika are well documented $[9,10]$. Three fruits component of chatuphalatika, T. chebula, T. bellerica and P. emblica, are formulated as a well-known ayurvedic herbal formulation named triphala. A number of publications on pharmacological evidences of triphala and its fruit constituents have been established. Triphala has been shown the antioxidant, hypolipidemic, antiobesogenic, hypoglycemic, anti-inflammatory, immunomodulatory and anticancer activities [11-14] and the prevention of dental caries [15]. Therefore, chatuphalatika should be considered as one of the potential herbal formulations for primary healthcare system and metabolic disease prevention.

Numerous studies characterized the pathological response of animal to a high-fat diet (HFD). Fat enriched diets feeding induce obesity and dyslipidemia in mice and rats [16]. The extracts of each fruit constituents of chatuphalatika have been reported to ameliorate body weight gain and/or improve blood lipid profile in animal models $[17,18]$. Thus, we hypothesized that chatuphalatika should be beneficial for the overweight and dyslipidemic management. The present study, we investigated the acute oral toxicity of chatuphalatika aqueous extract (CPT) in mice and then primarily tested for the lipid lowering effect in HFD- induced dyslipidemic mice.

\section{Methods}

\section{Plant materials and CPT preparation}

The fresh fruits of T. chebula, T. bellerica and P. emblica were collected from Doisaket district, Chiang Mai, Thailand. T. arjuna fruit was purchased from Tong In drugstore, Maha Sarakham, Thailand. All plant materials were identified by Assist.Prof. Wanida Caichampoo, Pharmaceutical Chemistry and Natural Product Research Unit, Faculty of Pharmacy, Mahasarakham University, Maha Sarakham, Thailand. The 
voucher specimens were deposited at Faculty of Pharmacy, Mahasarakham University, Maha Sarakham, Thailand. The seed of each fruit was removed and the seedless fruits were dried in hot air oven at $60^{\circ} \mathrm{C}$ for $48 \mathrm{~h}$. Each seedless dried fruit was blended to fine powder and sieved through mesh no.18. CPT was prepared in form of dried powder. Each $15 \mathrm{~g}$ of powder of T. chebula, T. bellerica, T. arjuna and P. emblica were mixed and boiled with $1 \mathrm{~L}$ of distilled water for $1 \mathrm{~h}$. The extracted solution was filtered through filter paper (Whatman Ò Filter Paper No.1) and then converted to powder by freeze dryer (ScanVac, Denmark).

\section{Total phenolic content determination}

Total phenolic content assay was performed by using a rapid microplate Folin-Ciocalteu method [19]. CPT sample solution was prepared in the concentration of $1 \mathrm{mg} / \mathrm{mL}$. Ten microliter of sample solution or gallic acid solution was mixed with $100 \mu \mathrm{L}$ of Folin-Ciocalteu working solution. Added $1 \mathrm{M}$ sodium carbonate in a volume of $80 \mu \mathrm{L}$ and mixed thoroughly. The reaction mixture was allowed to stand for 20 min at room temperature and the total phenolic content was determined by UV spectrophotometry (SPECTROstar Nano, BMG LABTECH, Germany) at $630 \mathrm{~nm}$. Linear correlation of gallic acid concentrations $(x)$ and absorbance $(y)$ was $y=1.0322 x+0.0588, R^{2}=0.998$. Total phenolic content of CPT was determined and represented as $\mathrm{mg}$ gallic acid equivalent (GAE) per $\mathrm{g}$ of extract.

\section{HPLC quantitative analysis of CPT}

Four phenolic compounds of the individual fruit constituent of chatuphalatika as gallic acid, ellagic acid, chebulinic acid and chebulagic acid were employed as chemical markers of CPT. Standard gallic acid, chebulinic acid and chebulagic acid (Sigma Aldrich Chemical Co., St. Louis, MO, USA) were dissolved in methanol (1 mg/mL). Stock solution of ellagic acid (Sigma Aldrich Chemical Co., St. Louis, MO, USA) was prepared in the concentration of $0.1 \mathrm{mg} / \mathrm{mL}$. All standard solutions were freshly prepared prior to use. Desired concentrations of chemical marker were subsequently diluted with methanol from the stock solutions. CPT was dissolved in methanol at a concentration of $2 \mathrm{mg} / \mathrm{mL}$ and then filtered through 0.45 $\mu \mathrm{m}$ PTFE syringe filter (RaphiLe Bioscience, China).

In this study, the condition of HPLC analysis method was slightly modified from our previous study [8]. Chromatographic system consisted of Shimadzu SCL-10A VP equipped with LC-10 AD binary pumps and SPD-M20A photodiode array detector (Kyoto, Japan). Mobile phase was a mixture of solvents: $0.05 \%$ trifluoroacetic acid in water (Solvent A) and (Solvent B). The separation was carried out in a linear gradient elution mode on an Eclipse XDB-C18 column with $5 \mu \mathrm{m}$ particle size, $4.6 \mathrm{~mm}$ internal diameter and $250 \mathrm{~mm}$ length as follows: 0-2 min, 5\% B; 2-4 min, 5-10\% B; 4-12 min, 10-15\% B; 12-26 min, 15-30\% B;26-30 min, 30-100\% B; 30-35 min, 100\% B; 35-40 min, 100-5\% B; 40-45 min, 5\% B. Mobile phase flow rate was $1.0 \mathrm{~mL} / \mathrm{min}$. Injection volume was $20 \mu \mathrm{L}$ and was introduced into the HPLC system in triplicate. The chromatographic runs were carried out at ambient temperature and the absorbance was detected at $270 \mathrm{~nm}$. The corresponding peaks of phytochemical markers were confirmed by retention times and spectrum comparing with standards. Under chromatographic conditions, the contents of chemical marker were calculated from calibration curves. 


\section{Acute oral toxicity study of CPT}

The acute toxicity study of CPT was performed in accordance with the Organisation for Economic Cooperation and Development (OECD) guidelines, test guideline 420.

\section{Animals}

Wistar rats of both sex ( $\mathrm{n}=30$, weighting $190-280 \mathrm{~g}, 8$ weeks old) were obtained from the National Laboratory Animal Center (Mahidol University, Nakornpathom, Thailand). The animals were housed under standard conditions (ambient temperature $24 \pm 2^{\circ} \mathrm{C}$, humidity $55 \pm 10 \%, 12$-h light/dark cycle (lights on from 7:00 $\mathrm{h}$ to 19:00 h)) and allowed free access to the standard rodent laboratory chow (No. CP082, Perfect Companion Group Co. Ltd., Thailand) and drinking water. They were acclimatized to these housing conditions for 1 week before the experiments. The experimental protocol was approved by the Institutional Animal Care and Use Committee, Mahasarakham University, Thailand (Approval no.00022558).

\section{CPT preparation and administration}

The CPT solution was freshly prepared before use. The extract was dissolved in the distilled water (DDW) and administered to the animals orally in the volume of $10 \mathrm{ml} / \mathrm{kg}$ body weight with the feeding needle.

\section{Experimental protocols}

The animals were divided into 3 groups of 10 animals per group with each having equal numbers of male and female animals. Each group of animals received a single oral-dose of either DDW or CPT in DDW ( 2,000 or $5,000 \mathrm{mg} / \mathrm{kg}$ body weight). The animals were fasted overnight (12 hours) before administration of drug and 4 hours after dosing. After administration of drug, the animals were observed for mortality and clinical signs of toxicity within the first four hours, and daily for a period of 14 days. On day 15 , the animals were subjected to blood collection for hematological analysis and necropsy. The body weight and food and water consumptions of the animals were recorded once daily throughout the experiment.

\section{Clinical observations}

The animals were observed at cage-side for clinical signs of toxicity including changes in skin, fur, eyes, mucous membranes, respiratory system, circulatory system, autonomic and central nervous systems, somatomotor activity and behavior pattern. They were carefully observed for tremors, convulsion, salivation, diarrhea, lethargy, sleep and coma.

\section{Hematological analysis}

The animals were anesthetized by an intraperitoneal injection of the thiopental sodium $(50 \mathrm{mg} / \mathrm{kg} \mathrm{BW}$; Jagsonpal Pharmaceuticals Ltd., Faridabad, Haryana, India) and the blood samples were collected via cardiac puncture in the K2-EDTA tubes (BD Vacutainer blood collection tube; Becton Dickinson, Franklin Lakes, New Jersey, USA). The white blood cells (WBC) count, neutrophils, lymphocytes, monocytes, 
eosinophils, basophils, red blood cells (RBC) count, hemoglobin, hematocrit, mean corpuscular volume $(\mathrm{MCV})$, mean corpuscular hemoglobin $(\mathrm{MCH})$, mean corpuscular hemoglobin concentration (MCHC) and platelet count were measured using V-53 reagent kit (Mindray, P.R. China) and BC-5300 Vet Auto Hematology Analyzer (Mindray, P.R. China).

\section{Necropsy}

The animals were euthanized by an intraperitoneal injection of overdose thiopental sodium and the internal organs including brain, heart, lungs, liver, stomach, spleen, kidneys, adrenal glands, bladder, testes, uterus and ovaries were harvested. The organs were weighed and observed for the gross lesions. The organ weight was used to calculate the relative organ weight by the following formula: Relative organ weight $=$ (organ weight $(\mathrm{g}) /$ body weight of the animal on sacrifice day $(\mathrm{g})) \times 100$.

\section{Lipid lowering study of CPT}

\section{Animals}

The thirty-six healthy male C57BL/6Mlac mice (weighting 20-25 g, 8 weeks old) were obtained from the National Laboratory Animal Center (Mahidol University, Nakornpathom, Thailand). The animals were housed under standard conditions as described in the acute oral toxicity study of the CPT. They were acclimatized to these housing conditions for 1 week before the experiments. The experimental protocol was approved by the Institutional Animal Care and Use Committee, Mahasarakham University, Thailand (Approval no.0002-2558).

\section{HFD}

The standard rodent laboratory chow was used herein as the normal diet (ND) containing $59.82 \%$ of energy from carbohydrates, $28.95 \%$ of energy from proteins and $11.23 \%$ of energy from fats. The HFD was prepared by adding additional ingredients into the ND. The final composition of the HFD consisted of the ND (33\%), casein (23\%), lard (33\%), cholesterol (1\%), vitamin \& mineral mixture (1\%), DL-methionine $(0.5 \%)$, butter $(3 \%)$, sucrose $(5 \%)$ and choline chloride $(0.5 \%)$. The HFD contained $19.09 \%$ of the energy from carbohydrates, $19.31 \%$ of the energy from proteins and $61.60 \%$ of the energy from fats. The energy content per gram of the ND and HFD were 3.69 and $5.29 \mathrm{kcal}$, respectively.

\section{Drug preparation and administration}

The CPT and atorvastatin (ATV) (Lipitor ${ }^{\circledR}$; Pfizer Inc, New York, NY, USA) were dissolved in the DDW and being administered to the animals by oral gavage in the volume of $10 \mathrm{ml} / \mathrm{kg}$ body weight. The drug administration was performed at the same time (10:00 $\mathrm{h}$ to $11: 00 \mathrm{~h})$ every day.

\section{Experimental protocols}


The animals were divided into two groups with the same average body weight. One group of animals was fed with the ND (ND group; $n=6$ ) and the other group of animals was fed with the HFD (HFD group; $\mathrm{n}=$ 30) for 16 weeks. After 10 weeks of feeding, the blood samples were collected from the animals in both groups and used for the lipid profile investigation. Then, the animals fed with the HFD were further divided into five groups with the same average body weight and were assigned to receive DDW (HFD + DDW group; $\mathrm{n}=6$ ), $10 \mathrm{mg} / \mathrm{kg}$ of ATV (HFD + ATV $10 \mathrm{mg} / \mathrm{kg}$ group; $\mathrm{n}=6), 250 \mathrm{mg} / \mathrm{kg}$ of CPT (HFD + CPT $250 \mathrm{mg} / \mathrm{kg}$ group; $\mathrm{n}=6$ ), $500 \mathrm{mg} / \mathrm{kg}$ of CPT (HFD + CPT $500 \mathrm{mg} / \mathrm{kg}$ group; $\mathrm{n}=6$ ) or $1,000 \mathrm{mg} / \mathrm{kg}$ of CPT $($ HFD + CPT 1,000 mg/kg group; $\mathrm{n}=6$ ) for 6 weeks (from week 11 to 16 of HFD feeding) while the animals fed with the ND received DDW (ND + DDW group; $n=6$ ) for 6 weeks (from week 11 to 16 of ND feeding). At the end of the feeding, the blood samples were collected from all animals for the lipid profile investigation. The body weight and food intake of the animals were measured once daily throughout the experiment. The caloric intake was calculated by multiplying the food intake $(\mathrm{g})$ by the energy content per gram of diet (kcal).

\section{Plasma lipid determination}

The blood samples were collected from the tail vein of the overnight fasted animals into the heparinized tubes and centrifuged at $6,000 \mathrm{rpm}$ for 10 minutes at $4^{\circ} \mathrm{C}$. The plasma was obtained and processed for the determination of the TC levels by the CHOD-POD enzymatic colorimetric method, the HDL-C levels by the GPO-POD enzymatic colorimetric method and the triglyceride (TRIG) levels by the direct enzymatic colorimetric method using the commercial kits (Mindray Bio Medical Co., Ltd., P.R. China) and BS-380 Automatic Biochemistry Analyzer (Mindray, P.R. China). The LDL-C levels was calculated using Friedewald's equation: LDL-C $=$ TC - HDL-C - TRIG/5 [20].

\section{Statistical analysis}

The statistical analysis was performed with SigmaPlot version-11 computer software (Systat software, San Jose, CA, USA). The results were reported as mean \pm SEM. The data was analyzed by Student's $t$-test, one-way ANOVA followed by the Student-Newman-Keuls test or two-way repeated measures ANOVA followed by the Student-Newman-Keuls test.

\section{Results}

\section{Phytochemical quantification of CPT}

The obtained CPT was dark brown dry powder and percentage of yielding was $21.89 \%$. Total phenolic content of CPT was $423.01 \pm 1.86 \mathrm{mg} \mathrm{GAE} / \mathrm{g}$ of extract. Figure 1 shows HPLC chromatogram of CPT and four phenolic compounds quantitatively analyzed as shown in Table 1. Gallic acid was a major phenolic compound of CPT with $137.1 \pm 5.42 \mathrm{mg} / \mathrm{g}$ of extract. The other phytochemical makers being identified as ellagic acid and ellagitannins group was totally found to be $141.4 \mathrm{mg} / \mathrm{g}$ of extract.

\section{Acute oral toxicity of CPT}


The single oral administration of CPT at the doses of 2,000 and $5,000 \mathrm{mg} / \mathrm{kg}$ did not produce any clinical signs of toxicity and mortality in both the male and female treated rats during 14-day observation period. The skin, fur, eyes, mucous membranes, respiratory system, circulatory system, autonomic and central nervous systems, somatomotor activity and behavior pattern of the treated rats were found to be normal. The tremors, convulsion, salivation, diarrhea, lethargy, sleep, coma and death did not occur in any of the treated rats. As there were no clinical signs of toxicity and mortality in both the tested doses, the $L_{50}$ value of CPT was found to be greater than $5,000 \mathrm{mg} / \mathrm{kg}$.

The body weight, and food and water consumptions of rats treated with CPT are shown in Figure 2 and Table 2. The body weight and body weight gain of the treated rats were not significantly different when compared with the control rats. No significant differences were observed in food and water consumptions. As shown in Table 3, all hematological parameter measured did not show statistically significant differences among the treated and control rats.

From the present study, no abnormal gross findings were observed in the necropsies of rats treated with $\mathrm{CPT}$. The relative organ weight recorded at necropsy of treated rats did not show a significant difference when compared with their respective control rats (Table 4).

\section{Lipid lowering effect of CPT}

\section{Effects of CPT on body weight and caloric intake in HFD-induced obese mice}

During the 16-week experiment, the body weight was measured every day and the food intake was measured every other day. The caloric intake was calculated daily as the grams of food intake multiplied by the caloric value of each diet. Figure 3B shows the cumulative caloric intake in the mice fed with HFD for 10 weeks. Compared with the mice in the ND group, the mice in the HFD group had higher cumulative caloric intake starting at 2 weeks and continuing through 10 weeks of feeding (week 2, $P=0.016$; week 3$10, P=<0.001$ ). After 10 weeks of feeding, the mice in the HFD group showed a $24.74 \%$ increase in the cumulative caloric intake when compared with the mice in the ND group. Along with increased in the cumulative caloric intake, the HFD-fed mice showed an increase in the cumulative body weight gain when compared with the ND-fed mice (Figure 3A). Starting from the 4 weeks until 10 weeks of feeding, the cumulative body weight gain was higher in the mice from the HFD group than the ND group (week 4, $P=$ 0.004; week 5, $P=0.005$; week $6, P=0.002$; week 7-10, $P=<0.001$ ). The cumulative body weight gain after 10 weeks of feeding was increased by $49.36 \%$ in the mice in the HFD group compared with the mice in the ND group.

The mice fed with HFD for 10 weeks became markedly obese as evidenced by the highest increase in the cumulative body weight gain (Figure 3A). The obese mice were then fed with the HFD supplemented with DDW, ATV $(10 \mathrm{mg} / \mathrm{kg})$ or CPT $(250,500$ or $1,000 \mathrm{mg} / \mathrm{kg})$ for 6 weeks. The effects of CPT on the cumulative body weight gain and the cumulative caloric intake in the HFD-induced obese mice are shown in Figure 4. Starting from the 3 weeks until 6 weeks of supplementation and compared with the normal mice (the mice fed with ND for 10 weeks) fed with ND supplemented with DDW, the obese mice fed with 
HFD supplemented with DDW had higher the cumulative body weight gain (week 3-6, $P=<0.001$ ) (Figure $4 \mathrm{~A}$ ) and the cumulative caloric intake (week 3, $P=0.005$; week 4-6, $P=<0.001$ ) (Figure 4B). The HFD-fed obese mice supplemented with $1,000 \mathrm{mg} / \mathrm{kg}$ CPT showed a reduction in the cumulative body weight gain when compared with the HFD-fed obese mice supplemented with DDW. These reductions were observed at 3 weeks after supplementation and continued throughout the supplementation period (week $3, P=$ 0.006; week 4-6, $P=<0.001$ ) (Figure 4A). However, during this time, there was no change in the cumulative caloric intake in the HFD-fed obese mice supplemented with $1,000 \mathrm{mg} / \mathrm{kg}$ CPT (week $3, P=0.994$; week 4, $P=0.934$; week 5, $P=0.400$; week 6, $P=0.477$ ) (Figure 4B).

\section{Effects of CPT on plasma lipid levels in HFD-induced obese mice}

The plasma lipid levels in the mice fed with HFD for 10 weeks are shown in Figure 5. The mice in the HFD group showed an increase in the plasma levels of TC $(P=<0.001)$, TRIG $(P=<0.001)$ and LDL-C $(P=$ $<0.001)$ and a decrease in the plasma levels of HDL-C $(P=<0.001)$ when compared with the mice in the ND group. These results indicated that the mice fed with HFD for 10 weeks developed dyslipidemia.

Then, the dyslipidemia mice were fed with HFD supplemented with DDW, ATV $(10 \mathrm{mg} / \mathrm{kg})$ or CPT (250, 500 or $1,000 \mathrm{mg} / \mathrm{kg})$ for 6 weeks. The elevated plasma levels of TC $(P=<0.001)$ (Figure 6A), TRIG $(P=$ $<0.001$ ) (Figure 6C) and LDL-C $(P=<0.001)$ (Figure 6D) and the reduced plasma levels of HDL-C $(P=$ $<0.001$ ) (Figure 6B) were observed in the HFD-fed dyslipidemia mice supplemented with DDW compared with the ND-fed normal mice supplemented with DDW. ATV $(10 \mathrm{mg} / \mathrm{kg})$ supplementation decreased the plasma levels of TC $(P=<0.001)$ (Figure 6A), TRIG $(P=<0.001)$ (Figure 6C) and LDL-C $(P=<0.001)$ (Figure 6D) and increased the plasma levels of HDL-C $(P=<0.001)$ (Figure 6B) when compared with DDW supplementation in HFD-fed dyslipidemia mice. Compared with the HFD-fed dyslipidemia mice supplemented with DDW, the HFD-fed dyslipidemia mice supplemented with $250 \mathrm{mg} / \mathrm{kg}$ CPT exhibited decreased the plasma LDL-C levels $(P=<0.001)$ (Figure 6D) and increased the plasma HDL-C levels $(P=$ $<0.001$ ) (Figure 6B). Supplementation with 500 or $1,000 \mathrm{mg} / \mathrm{kg}$ CPT compared with DDW decreased the plasma levels of TC $(P=<0.001)$ (Figure 6A) and LDL-C $(P=<0.001)$ (Figure 6D) and increased the plasma levels of HDL-C $(P=<0.001)$ (Figure 6B) in the HFD-fed dyslipidemia mice.

\section{Discussion}

Obesity is an excessive body fat accumulation resulting from an imbalance of food intake and energy expenditure. Causes of obesity are regarded complex including diets, habits and genetic factors. Major complications of obesity are coronary artery disease, hypertension, type 2 diabetes mellitus, respiratory disorders and dyslipidemia [21]. Beside synthetic drugs, the development of natural medicines for preventing and ameliorating obesity have been widely explored [22-24]. The individual fruit constituents of chatuphalatika have been proclaimed for their biological activities on overweight complication. Water extract of $T$. bellerica fruit demonstrated a preventive effect on obesity, insulin resistance and hyperlipidemia [25]. Terminalia chebula, T. bellerica and P. emblica fruits showed body weight and body fat reduction effects in mice $[18,26]$. The phytochemical investigation demonstrated gallic acid, ellagic 
acid, chebulinic acid, chebugalic acid and corilagin were the major phytoconstituents of Terminalia and Phylanthus fruits $[27,28]$. These phenolic compounds have been reported to be responsible for an alleviation of fat accumulation [29,30]. Our study provides the first evidence for safety, anti-dyslipidemic and anti-obesity efficacy of chatuphalatika formula extract in experimental animals.

The acute toxicity evaluation of a test substance is a necessary step to determine the adverse effects that might occur within a short time (24 hours) after administration of test substance [31]. In the present study, the evaluation of acute oral toxicity of CPT was carried out according to the OECD guideline 420 which was developed to reduce the suffering and the number of animals used in general acute toxicity test [32]. The results showed that the rats receiving a single oral administration of 2,000 and $5,000 \mathrm{mg} / \mathrm{kg}$ of CPT survived and demonstrated normal behavior, food and water consumptions, body weight, organ size and morphology, and hematological parameters. The results obtained revealed safety of orally given CPT at the doses up to $5,000 \mathrm{mg} / \mathrm{kg}$.

The HFD-induced obese C57BL/6 mice is a common animal model for studying the obesity and obesityrelated metabolic syndrome [33]. In the present study, 10 weeks of HFD feeding in C57BL/6 mice resulted in the increase in body weight and the changes in blood lipid profiles shown as reducing HDL-C and elevating TC, TRIG and LDL-C. These conditions are similar to lipid profiles found in the patients of dyslipidemia associated with obesity. After 6 weeks of CPT supplementation to HFD fed dyslipidemia mice, we observed that CPT at the dose of $250 \mathrm{mg} / \mathrm{kg}$ increased plasma HDL-C levels and decreased plasma LDL-C levels, while CPT at the doses of 500 and 1,000 mg/kg increased plasma HDL-C levels and decreased both plasma TC and LDL-C levels. However, the CPT supplementation at all doses tested (250, 500 and $1,000 \mathrm{mg} / \mathrm{kg}$ ) did not reduce the elevation of plasma TRIG levels in the HFD fed dyslipidemia mice. Interestingly, the HFD fed dyslipidemia mice supplemented with 1,000 mg/kg CPT showed the body weight gain reduction without calorie intake alteration. Thus, CPT at high dose could reduce both dyslipidemia and body weight gain, whereas at low dose CPT could only reduce dyslipidemia. Therefore, it is highly suggestive that CPT may be used as a supplement and/or functional food to relieve dyslipidemia especially those in association with obesity and metabolic syndrome.

Phenolics are a large group of secondary metabolites naturally abundant in plants. The growing of scientific evidences supporting their anti-obesogenic properties are convincing [34]. CPT contained approximately $70 \%$ polyphenolic compounds predominantly gallic acid, ellagic acid, chebulagic acid and a small amount of chebulinic acid. These phenolic compounds have been reported to alleviate the fat accumulation $[29,30]$. There are promising dietary polyphenolics that could modulate the development of obesity associated with metabolic consequences. Previous study reported that gallic acid $50 \mathrm{mg} / \mathrm{kg}$ supplementation for 10 weeks showed the improvement of lipid profile in HFD induced hypercholesterolemic rats, reduced the body weight and adipose tissue weight [35]. Intake of ellagic acid $35.6 \mathrm{mg} / \mathrm{kg}$ for 8 weeks resulted in a significant reduction of body weight and total abdominal fat in HFDfed rats [36]. Although, the anti-obesity mechanisms of CPT are not yet elucidated, it is likely that these phenolics were the active compounds exerting body weight loss and hypolipidemic effects of chatuphalatika through multitarget of actions. The lipid digestion and absorption are the important steps 
relevant to the development of obesity. Dietary fat is not absorbed in gastrointestinal tract until the catalytic enzyme is action. Makihara et al. [25] investigated the preventive effect of $T$. bellerica on obesity and clarified that gallic acid was the active component primarily responsible for inhibition of pancreatic lipase activity. Moreover, the in vitro studies demonstrated that gallic acid and ellagic acid can delay cholesterol absorption through inhibiting of pancreatic cholesterol esterase, binding of bile acids and reducing the solubility of cholesterol in micelles [37,38].

The cause of obesity is related to adipocyte development and lipid accumulation. 3T3-L1 cell line is an in vitro model to study the molecular mechanisms of adipogenesis [39]. The in vitro study demonstrated gallic acid had the high inhibition of 3T3-L1 pre-adipocyte cell growth [40]. Gallic acid has been reported to suppress abdominal fat through upregulated of peroxisome proliferator-activated receptor-a (PPPARa) expression, increased expression low-density lipoprotein receptor on hepatocytes, decreased proprotein convertase subtilisin/kexin type 9 (PCSK9) levels, increased activity of hepatic lipolytic and decreased lipogenic enzymes [41]. Ellagic acid was identified to response for suppress adipogenesis by decreasing the expression of adipogenic genes [42].

\section{Conclusions}

In summary, our study demonstrated that the CPT was considerably safe and it was beneficial to improve blood lipid profiles in the HFD induced dyslipidemia mice. The CPT oral administration ameliorated the body weight gain without limit caloric intake. The gallic acid and ellagitannins were the active compounds contributed to anti-dyslipidemic and anti-obesity effects of this herbal formula.

\section{List Of Abbreviations}

ATV: atorvastatin; CPT: Chatuphalatika aqueous extract; CVD: Coronary vascular disease; DDW: distilled water; GAE: gallic acid equivalent; HDL-C: high-density lipoprotein cholesterol; HFD: high-fat diet; HMGCoA: 3-hydroxy-3-methyl-glutaryl-coenzyme A; LDL-C: low-density lipoprotein cholesterol; $\mathrm{MCH}$ : mean corpuscular hemoglobin; MCHC: mean corpuscular hemoglobin concentration; MCV: mean corpuscular volume; ND: normal diet; OECD: Organisation for Economic Co-operation and Development; PCSK9: proprotein convertase subtilisin/kexin type 9; PPPARa: peroxisome proliferator-activated receptor-a; RBC: red blood cell; TC: total cholesterol; TRIG: triglyceride; VLDL-C: very low density lipoprotein cholesterol; WBC: white blood cells

\section{Declarations}

\section{Ethics approval and consent to participate}

The animal experimental protocol was approved by the Institutional Animal Care and Use Committee, Mahasarakham University, Thailand. 


\section{Consent for publication}

Not applicable

\section{Availability of data and materials}

Not applicable

\section{Competing interests}

The authors declare that they have no competing interests.

\section{Funding}

This work was financially supported by Faculty of Pharmacy, Mahasarakham University, Thailand and Thailand Research Fund (TRG5780201).

\section{Acknowledgements}

Not applicable

\section{Authors' contributions}

PR designed and conceived the study; prepared plant extract and performed chemical experiments. SM performed the animal experiments. PR and SM wrote the main manuscript text, analyzed the data and prepared the figures. MT made valuable revision of the manuscript. All authors read and approved the final manuscript.

\section{Author details}

${ }^{1}$ Department of Physiology, Faculty of Medicine, Chiang Mai University, Chiang Mai 50200, Thailand. ${ }^{2}$ Faculty of Pharmaceutical Sciences, Burapha University, Chonburi 20131, Thailand. ${ }^{3}$ Pharmaceutical Chemistry and Natural Product Research Unit, Faculty of Pharmacy, Mahasarakham University, Maha Sarakham 44150, Thailand. 


\section{References}

1. Sanchis-Gomar F, Perez-Quilis C, Leischik R, Lucia A. Epidemiology of coronary heart disease and acute coronary syndrome. Ann Transl Med. 2016;4(13):256.

2. Riaz H, Khan MS, Siddiqi TJ, Usman MS, Shah N, Goyal A, et al. Association between obesity and cardiovascular outcomes: a systematic review and meta-analysis of mendelian randomization studies. JAMA Netw open. 2018;1(7):e183788-8.

3. Chooi YC, Ding C, Magkos F. The epidemiology of obesity. Metabolism. 2019;92:6-10.

4. Ortega FB, Lavie CJ, Blair SN. Obesity and cardiovascular disease. Circ Res. 2016;118(11):1752-70.

5. Howe E, Keiwkarnka BKM. Traditional medicine and medicinal plants: utilization, policy and research in Thailand. J Public Heal Dev. 2004;2(1):101-18.

6. Peltzer K, Pengpid S, Puckpinyo A, Yi S, Anh LV. The utilization of traditional, complementary and alternative medicine for non-communicable diseases and mental disorders in health care patients in Cambodia, Thailand and Vietnam. BMC Complement Altern Med. 2016;16:92.

7. Saralamp P, Soonthornchareonnon NAN. Handbook of medicinal herbs in mational essential drug list. 3rd ed. Bangkok: Concept Medicus; 2011.

8. Sato VH, Sungthong B, Rinthong P-O, Nuamnaichati N, Mangmool S, Chewchida S, et al. Pharmacological effects of Chatuphalatika in hyperuricemia of gout. Pharm Biol. 2018;56(1):76-85.

9. Baliga MS, Meera S, Mathai B, Rai MP, Pawar V, Palatty PL. Scientific validation of the ethnomedicinal properties of the Ayurvedic drug Triphala: A review. Chin J Integr Med. 2012;18(12):946-54.

10. Amalraj A, Gopi S. Medicinal properties of Terminalia arjuna (Roxb.) Wight \& Arn.: A review. J Tradit Complement Med. 2017;7(1):65-78.

11. Naik GH, Priyadarsini KI, Bhagirathi RG, Mishra B, Mishra KP, Banavalikar MM, et al. In vitro antioxidant studies and free radical reactions of triphala, an ayurvedic formulation and its constituents. Phyther Res. 2005;19(7):582-6.

12. Saravanan S, Srikumar R, Manikandan S, Jeya PN, Sheela DR. Hypolipidemic effect of Triphala in experimentally induced hypercholesteremic rats. Yakugaku Zasshi. 2007;127(2):385-8.

13. Prakash S, Shelke A. Role of Triphala in dentistry. J Indian Soc Periodontol. 2014;18(2):132-5.

14. Peterson CT, Denniston K, Chopra D. Therapeutic uses of Triphala in Ayurvedic medicine. J Altern Complement Med. 2017;23(8):607-14.

15. Kalaiselvan S, Rasool MK. Triphala herbal extract suppresses inflammatory responses in LPSstimulated RAW 264.7 macrophages and adjuvant-induced arthritic rats via inhibition of NF-KB pathway. J Immunotoxicol. 2016;13(4):509-25.

16. Fraulob JC, Ogg-Diamantino R, Fernandes-Santos C, Aguila MB, Mandarim-de-Lacerda CA. A mouse model of metabolic syndrome: insulin resistance, fatty liver and non-alcoholic fatty pancreas disease (NAFPD) in C57BL/6 mice fed a high fat diet. J Clin Biochem Nutr. 2010;46(3):212-23. 
17. Maruthappan V, Shree KS. Hypolipidemic activity of haritaki (Terminalia chebula) in atherogenic diet induced hyperlipidemic rats. J Adv Pharm Technol Res. 2010;1(2):229-35.

18. Gurjar S, Pal AKS. Triphala and its constituents ameliorate visceral adiposity from a high-fat diet in mice with diet-induced obesity. Altern Ther Heal Med. 2012;18(6):38-45.

19. Attard E. A rapid microtitre plate Folin-Ciocalteu method for the assessment of polyphenols. Cent Eur J Biol. 2013;8(1):48-53.

20. Friedewald WT, Levy RI, Fredrickson DS. Estimation of the concentration of low-density lipoprotein cholesterol in plasma, without use of the preparative ultracentrifuge. Clin Chem. 1972;18(6):499502.

21. Klop B, Elte JWF, Cabezas MC. Dyslipidemia in obesity: mechanisms and potential targets. Nutrients. 2013;5(4):1218-40.

22. Sun N-N, Wu T-Y, Chau C-F. Natural dietary and herbal products in anti-obesity treatment. Molecules. 2016;21(10):1351.

23. Liu Y, Sun M, Yao H, Liu Y, Gao R. Herbal medicine for the treatment of obesity: an overview of scientific evidence from 2007 to 2017. Kismali G, editor. Evidence-Based Complement Altern Med. 2017.

24. Park Y-J, Lee G-S, Cheon S-Y, Cha Y-Y, An H-J. The anti-obesity effects of Tongbi-san in a high-fat diet-induced obese mouse model. BMC Complement Altern Med. 2019;19(1):1.

25. Makihara $H$, Shimada T, Machida E, Oota M, Nagamine R, Tsubata $M$, et al. Preventive effect of Terminalia bellirica on obesity and metabolic disorders in spontaneously obese type 2 diabetic model mice. J Nat Med. 2012;66(3):459-67.

26. Reddy MM, Dhas Devavaram J, Dhas J, Adeghate E, Starling Emerald B. Anti-hyperlipidemic effect of methanol bark extract of Terminalia chebula in male albino Wistar rats. Pharm Biol. 2015;53(8):1133-40.

27. Dhanani T, Shah S, Kumar S. A validated high-performance liquid chromatography method for determination of tannin-related marker constituents gallic acid, corilagin, chebulagic acid, ellagic acid and chebulinic acid in four Terminalia species from India. J Chromatogr Sci. 2014;53(4):62532.

28. Yang B, Liu P. Composition and biological activities of hydrolyzable tannins of fruits of Phyllanthus emblica. J Agric Food Chem. 2014;62(3):529-41.

29. Makihara H, Koike Y, Ohta M, Horiguchi-Babamoto E, Tsubata M, Kinoshita K, et al. Gallic acid, the active ingredient of Terminalia bellirica, enhances adipocyte differentiation and adiponectin secretion. Biol Pharm Bull. 2016;39(7):1137-43.

30. Huang D-W, Chang W-C, Yang H-J, Wu JS-B, Shen S-C. Gallic acid alleviates hypertriglyceridemia and fat accumulation via modulating glycolysis and lipolysis pathways in perirenal adipose tissues of rats fed a high-fructose diet. Int J Mol Sci. 2018;19(1):254.

31. Duffus JH, Templeton DMNM. Concepts in toxicology. Cambridge: RSC Publishing; 2009. 
32. OECD guidelines for testing of chemicals Sect. 4: health effects. In: Organisation for Economic Cooperation and Development (OECD). Paris: OECD publishing; 2002.

33. Fuchs T, Loureiro M, de P, Macedo, Nocca LE, Nedelcu D, Costa-Casagrande M TA. Modelos animais na síndrome metabólica.. Vol. 45, Revista do Colégio Brasileiro de Cirurgiões. scielo ; 2018.

34. Rodríguez-Pérez C, Segura-Carretero A, del Mar Contreras M. Phenolic compounds as natural and multifunctional anti-obesity agents: A review. Crit Rev Food Sci Nutr. 2019;59(8):1212-29.

35. Hsu C-L, Yen G-C. Effect of gallic acid on high fat diet-induced dyslipidaemia, hepatosteatosis and oxidative stress in rats. Br J Nutr. 2007;98(4):727-35.

36. Panchal SK, Ward L, Brown L. Ellagic acid attenuates high-carbohydrate, high-fat diet-induced metabolic syndrome in rats. Eur J Nutr. 2013;52(2):559-68.

37. Ngamukote S, Mäkynen K, Thilawech T, Adisakwattana S. Cholesterol-lowering activity of the major polyphenols in grape seed. Molecules. 2011;16(6):5054-61.

38. Lei F, Zhang XN, Wang W, Xing DM, Xie WD, Su H, et al. Evidence of anti-obesity effects of the pomegranate leaf extract in high-fat diet induced obese mice. Int J Obes. 2007;31(6):1023-9.

39. Ntambi JM, Young-Cheul K. Adipocyte Differentiation and Gene Expression. J Nutr. 2000;130(12):3122S-3126S.

40. Furuyashiki T, Nagayasu H, Aoki Y, Bessho H, Hashimoto T, Kanazawa K, et al. Tea catechin suppresses adipocyte differentiation accompanied by Down-regulation of PPARY2 and C/EBPa in 3T3-L1 cells. Biosci Biotechnol Biochem. 2004;68(11):2353-9.

41. Variya BC, Bakrania AK, Chen Y, Han J, Patel SS. Suppression of abdominal fat and antihyperlipidemic potential of Emblica officinalis: Upregulation of PPARs and identification of active moiety. Biomed Pharmacother. 2018;108:1274-81.

42. Kang I, Buckner T, Shay NF, Gu L, Chung S. Improvements in metabolic health with consumption of ellagic acid and subsequent conversion into urolithins: Evidence and Mechanisms. Adv Nutr. 2016;7(5):961-72.

\section{Tables}

Table 1 The content of phytochemical markers in CPT.

Chemical markers Content of markers (mg/g extract)

Gallic acid

$137.1 \pm 5.42$

Ellagic acid and ellagitannins

141.1

Ellagic acid

$5.2 \pm 0.40$

Chebulagic acid

$62.6 \pm 4.17$

Chebulinic acid

$73.6 \pm 2.35$ 
Table 2. The effects of Chatuphalatika aqueous extract on body weight gain and food and water consumptions in male and female rats in acute toxicity study.

\begin{tabular}{lllll}
\hline \multirow{2}{*}{ Parameters } & Sex & \multicolumn{2}{l}{ Groups } & \\
\cline { 3 - 5 } & & Control & CPT 2,000 mg/kg & CPT 5,000 mg/kg \\
& & $(\mathbf{n}=10)$ & $(\mathbf{n}=10)$ & $(\mathbf{n}=10)$ \\
\hline Initial body weight $(\mathrm{g})$ & Male & $277.67 \pm 2.54$ & $274.67 \pm 1.76$ & $276.00 \pm 3.10$ \\
& Female & $195.67 \pm 1.14$ & $197.67 \pm 2.06$ & $195.83 \pm 3.46$ \\
Final body weight (g) & Male & $315.50 \pm 3.77$ & $319.17 \pm 3.42$ & $309.67 \pm 12.10$ \\
& Female & $212.17 \pm 2.91$ & $217.67 \pm 3.16$ & $216.83 \pm 4.01$ \\
Body weight gain (\%) & Male & $13.63 \pm 0.95$ & $16.22 \pm 1.28$ & $12.07 \pm 3.47$ \\
& Female & $8.43 \pm 1.36$ & $10.15 \pm 1.54$ & $10.73 \pm 0.81$ \\
Food intake (g/day) & Male & $114.00 \pm 2.50$ & $122.00 \pm 2.89$ & $116.79 \pm 4.50$ \\
& Female & $79.61 \pm 2.49$ & $81.00 \pm 2.07$ & $87.75 \pm 4.10$ \\
Water intake (ml/day) & Male & $214.39 \pm 9.16$ & $200.21 \pm 5.20$ & $222.00 \pm 7.76$ \\
& Female & $138.11 \pm 3.95$ & $142.43 \pm 2.40$ & $152.25 \pm 6.34$ \\
\hline
\end{tabular}

The rats were treated with a single oral administration of the Chatuphalatika aqueous extract $(2,000$ or $5,000 \mathrm{mg} / \mathrm{kg}$ ) or vehicle. They were weighed once prior to administration of the dosing and daily thereafter until the end of the 14-day experimental period. The food and water consumptions of the rats were record once daily throughout the experiment. The data are expressed as mean \pm SEM. Abbreviations: CPT, Chatuphalatika aqueous extract.

Table 3. The effects of Chatuphalatika aqueous extract on hematological parameters in male and female rats in acute toxicity study. 
Parameters

Groups

Sex

Control

CPT $2,000 \mathrm{mg} / \mathrm{kg}$ CPT $5,000 \mathrm{mg} / \mathrm{kg}$

$(n=10) \quad(n=10) \quad(n=10)$

\begin{tabular}{lllll}
\hline Hemoglobin $(\mathrm{g} / \mathrm{dl})$ & Male & $15.55 \pm 0.20$ & $15.29 \pm 0.52$ & $16.40 \pm 0.40$
\end{tabular}

Female $14.92 \pm 0.10 \quad 14.78 \pm 0.11 \quad 15.07 \pm 0.12$

$\operatorname{RBC}\left(10^{6} / \mu \mathrm{l}\right)$

Male $\quad 8.74 \pm 0.10$

$7.93 \pm 0.32$

$8.44 \pm 0.26$

Female $7.95 \pm 0.08$

$7.87 \pm 0.07$

$7.90 \pm 0.10$

Hematocrit (\%)

Male

$46.75 \pm 0.72$

$44.25 \pm 1.79$

$48.13 \pm 1.04$

Female $\quad 43.58 \pm 0.35$

$43.23 \pm 0.33$

$44.10 \pm 0.43$

$\operatorname{MCV}(f L)$

Male

$54.10 \pm 0.59$

$55.85 \pm 0.73$

$56.53 \pm 0.75$

Female $\quad 54.80 \pm 0.38$

$54.92 \pm 0.65$

$55.92 \pm 0.61$

$\mathrm{MCH}(\mathrm{pg})$

Male $\quad 18.38 \pm 0.15$

$18.70 \pm 0.21$

$18.88 \pm 0.21$

Female $\quad 18.77 \pm 0.18$

$18.78 \pm 0.23$

$19.35 \pm 0.20$

$\mathrm{MCHC}(\mathrm{g} / \mathrm{dl})$

Male

$33.25 \pm 0.12$

$33.47 \pm 0.25$

$34.08 \pm 0.32$

Female $34.22 \pm 0.18$

$34.20 \pm 0.13$

$34.63 \pm 0.18$

$\operatorname{WBC}\left(10^{3} / \mu \mathrm{l}\right)$

Male $\quad 10.46 \pm 0.68$

$8.23 \pm 0.79$

$9.30 \pm 0.38$

Female $\quad 6.58 \pm 0.54$

$6.38 \pm 0.49$

$5.22 \pm 0.37$

Neutrophils (\%)

Male $\quad 26.32 \pm 2.81$

$20.10 \pm 2.69$

$20.97 \pm 2.02$

Female $25.18 \pm 3.81$

$19.13 \pm 3.80$

$21.33 \pm 2.02$

Eosinophils (\%)

Male $\quad 1.83 \pm 0.29$

$2.65 \pm 0.53$

$1.70 \pm 0.31$

Female $\quad 1.80 \pm 0.30$

$2.57 \pm 0.76$

$1.83 \pm 0.55$

Lymphocytes (\%)

Male

$62.50 \pm 2.20$

$67.35 \pm 2.33$

$72.35 \pm 1.79$

Female $\quad 62.02 \pm 2.73$

$66.03 \pm 2.22$

$70.33 \pm 1.37$

Monocytes (\%)

Male

$9.08 \pm 1.03$

$8.93 \pm 1.36$

$4.87 \pm 1.29$

Female $10.97 \pm 1.46$

$12.23 \pm 1.63$

$6.50 \pm 1.58$

Basophils (\%)

Male

$0.27 \pm 0.06$

$0.97 \pm 0.83$

$0.12 \pm 0.05$

Female

$0.03 \pm 0.03$

$0.03 \pm 0.03$

$0.00 \pm 0.00$

Platelet $\left(10^{3} / \mu \mathrm{l}\right)$

Male $\quad 893.00 \pm 9.87$

$735.00 \pm 72.65$

$753.17 \pm 82.81$

Female $\quad 861.00 \pm 48.89$

$887.50 \pm 33.76$

$854.67 \pm 83.67$ 
The rats were treated with a single oral administration of the Chatuphalatika aqueous extract $(2,000$ or $5,000 \mathrm{mg} / \mathrm{kg}$ ) or vehicle. At 15 days after dosing, the rats were anesthetized and the blood samples were taken from the rat heart and analyzed for various hematological parameters. The data are expressed as mean \pm SEM. Abbreviations: CPT, Chatuphalatika aqueous extract; RBC, red blood cell; MCV, mean corpuscular volume; $\mathrm{MCH}$, mean corpuscular hemoglobin; $\mathrm{MCHC}$, mean corpuscular hemoglobin concentration; WBC, white blood cells.

Table 4. The effects of Chatuphalatika aqueous extract on relative organ weights in male and female rats in acute toxicity study. 


\begin{tabular}{|c|c|c|c|c|}
\hline \multirow{3}{*}{ Organs } & \multirow{3}{*}{ Sex } & \multicolumn{3}{|l|}{ Groups } \\
\hline & & Control & CPT $2,000 \mathrm{mg} / \mathrm{kg}$ & CPT $5,000 \mathrm{mg} / \mathrm{kg}$ \\
\hline & & $(n=10)$ & $(n=10)$ & $(n=10)$ \\
\hline \multirow[t]{2}{*}{ Brain } & Male & $0.486 \pm 0.013$ & $0.507 \pm 0.008$ & $0.472 \pm 0.011$ \\
\hline & Female & $0.732 \pm 0.020$ & $0.736 \pm 0.010$ & $0.734 \pm 0.019$ \\
\hline \multirow[t]{2}{*}{ Heart } & Male & $0.330 \pm 0.011$ & $0.345 \pm 0.014$ & $0.334 \pm 0.007$ \\
\hline & Female & $0.407 \pm 0.018$ & $0.396 \pm 0.013$ & $0.392 \pm 0.026$ \\
\hline \multirow[t]{2}{*}{ Lungs } & Male & $0.702 \pm 0.042$ & $0.773 \pm 0.035$ & $0.825 \pm 0.034$ \\
\hline & Female & $0.790 \pm 0.041$ & $0.865 \pm 0.040$ & $0.985 \pm 0.086$ \\
\hline \multirow[t]{2}{*}{ Liver } & Male & $3.877 \pm 0.171$ & $4.336 \pm 0.150$ & $4.448 \pm 0.188$ \\
\hline & Female & $4.176 \pm 0.102$ & $4.223 \pm 0.161$ & $4.488 \pm 0.049$ \\
\hline \multirow[t]{2}{*}{ Stomach } & Male & $1.039 \pm 0.079$ & $0.927 \pm 0.070$ & $0.985 \pm 0.035$ \\
\hline & Female & $1.121 \pm 0.069$ & $1.133 \pm 0.026$ & $1.183 \pm 0.053$ \\
\hline \multirow[t]{2}{*}{ Spleen } & Male & $0.214 \pm 0.009$ & $0.212 \pm 0.011$ & $0.224 \pm 0.010$ \\
\hline & Female & $0.263 \pm 0.017$ & $0.274 \pm 0.012$ & $0.289 \pm 0.031$ \\
\hline \multirow[t]{2}{*}{ Right kidney } & Male & $0.338 \pm 0.013$ & $0.338 \pm 0.006$ & $0.341 \pm 0.008$ \\
\hline & Female & $0.337 \pm 0.018$ & $0.357 \pm 0.008$ & $0.360 \pm 0.018$ \\
\hline \multirow[t]{2}{*}{ Left kidney } & Male & $0.319 \pm 0.010$ & $0.322 \pm 0.004$ & $0.336 \pm 0.013$ \\
\hline & Female & $0.335 \pm 0.010$ & $0.327 \pm 0.011$ & $0.334 \pm 0.007$ \\
\hline \multirow[t]{2}{*}{ Right adrenal gland } & Male & $0.012 \pm 0.001$ & $0.011 \pm 0.004$ & $0.008 \pm 0.001$ \\
\hline & Female & $0.017 \pm 0.002$ & $0.016 \pm 0.004$ & $0.011 \pm 0.001$ \\
\hline \multirow[t]{2}{*}{ Left adrenal gland } & Male & $0.011 \pm 0.000$ & $0.011 \pm 0.004$ & $0.008 \pm 0.001$ \\
\hline & Female & $0.016 \pm 0.001$ & $0.020 \pm 0.003$ & $0.016 \pm 0.001$ \\
\hline \multirow[t]{2}{*}{ Bladder } & Male & $0.031 \pm 0.005$ & $0.040 \pm 0.004$ & $0.041 \pm 0.005$ \\
\hline & Female & $0.047 \pm 0.009$ & $0.043 \pm 0.006$ & $0.037 \pm 0.006$ \\
\hline Right testis & Male & $0.498 \pm 0.008$ & $0.499 \pm 0.009$ & $0.493 \pm 0.018$ \\
\hline Left testis & Male & $0.522 \pm 0.015$ & $0.499 \pm 0.019$ & $0.492 \pm 0.011$ \\
\hline Uterus & Female & $0.246 \pm 0.016$ & $0.201 \pm 0.023$ & $0.225 \pm 0.025$ \\
\hline Right ovary & Female & $0.047 \pm 0.014$ & $0.045 \pm 0.008$ & $0.045 \pm 0.004$ \\
\hline
\end{tabular}


The rats were treated with a single oral administration of the Chatuphalatika aqueous extract $(2,000$ or $5,000 \mathrm{mg} / \mathrm{kg}$ ) or vehicle. At 15 days after dosing, the rats were euthanized after blood taken and the internal organs were collected, observed for the gross lesions and weighed to calculate the relative organ weights. The relative organ weight was calculated as organ weight $(\mathrm{g}) /$ body weight $(\mathrm{g}) \times 100$. The data are expressed as mean \pm SEM. Abbreviations: CPT, Chatuphalatika aqueous extract.

\section{Figures}

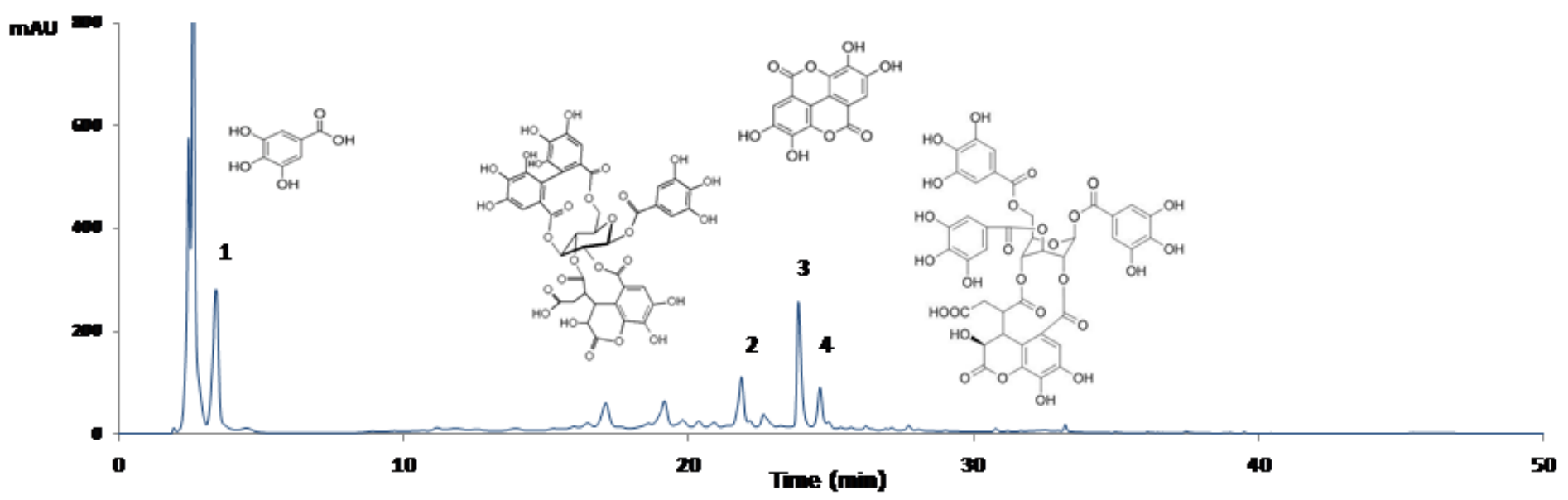

\section{Figure 1}

HPLC chromatogram of CPT at wavelength $270 \mathrm{~nm}$ : gallic acid (1), chebulagic acid (2), ellagic acid (3) and chebulinic acid (4). 



\section{Figure 2}

The effects of Chatuphalatika aqueous extract on body weight in male and female rats in acute toxicity study. The rats were treated with a single oral administration of the Chatuphalatika aqueous extract $(2,000$ or $5,000 \mathrm{mg} / \mathrm{kg})$ or vehicle. They were weighed once prior to administration of the dosing and daily thereafter until the end of the 14-day experimental period. The data are expressed as mean $\triangle \mathrm{SEM}(\mathrm{n}=5$ per group). Abbreviations: CPT, Chatuphalatika aqueous extract. 



Figure 3

The effects of the high-fat diet on the cumulative body weight gain (A) and cumulative caloric intake (B) in the mice. The mice were fed with the normal diet or high-fat diet for 10 weeks. The body weight and food intake of the mice were measured once daily throughout the experiment. The caloric intake was calculated by multiplying the food intake $(\mathrm{g})$ by the energy content per gram of diet ( $\mathrm{kcal})$. The data are expressed as mean \pm SEM ( $n=6$ in ND group, $n=30$ in HFD group). $\# P<0.05, \# \# P<0.01$, \#\#\#P<0.001 vs. ND group (two-way repeated measures ANOVA followed by the Student-Newman-Keuls test). Abbreviation: ND, normal diet; HFD, high-fat diet. 

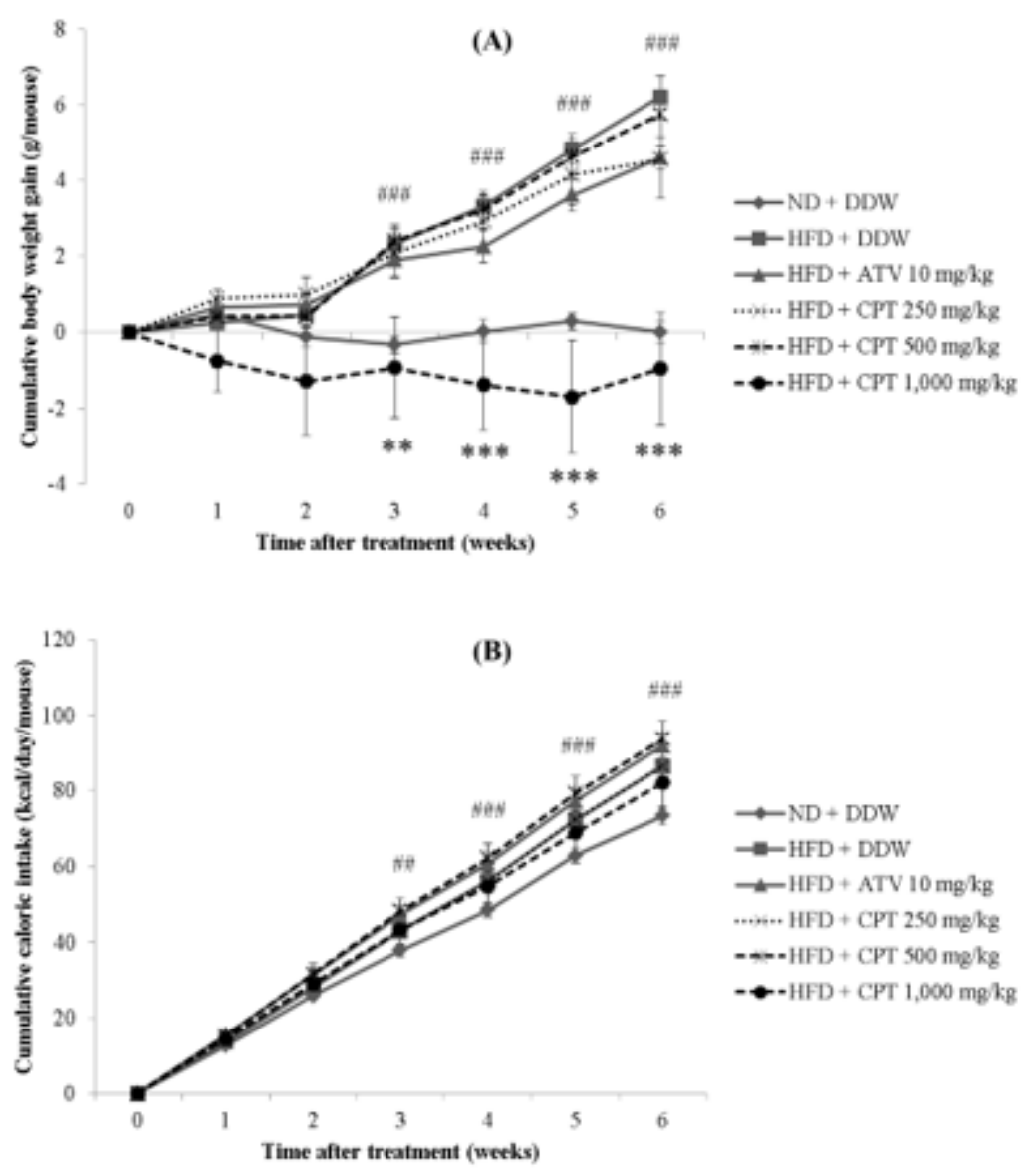

\section{Figure 4}

The effects of the Chatuphalatika aqueous extract on the cumulative body weight gain (A) and cumulative caloric intake (B) in the high-fat diet induced obese mice. The high-fat diet induced obese mice were fed the high-fat diet supplemented with the Chatuphalatika aqueous extract, atorvastatin or distilled water for 6 weeks. The body weight and food intake of the high-fat diet induced obese mice were measured once daily throughout the experiment. The caloric intake was calculated by multiplying the food intake $(\mathrm{g})$ by the energy content per gram of diet $(\mathrm{kcal})$. The data are expressed as mean \pm SEM $(\mathrm{n}=$

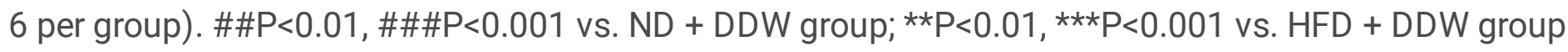
(two-way repeated measures ANOVA followed by the Student-Newman-Keuls test). Abbreviations: ND, normal diet; HFD, high-fat diet; DDW, distilled water; ATV, atorvastatin; CPT, Chatuphalatika aqueous extract. 


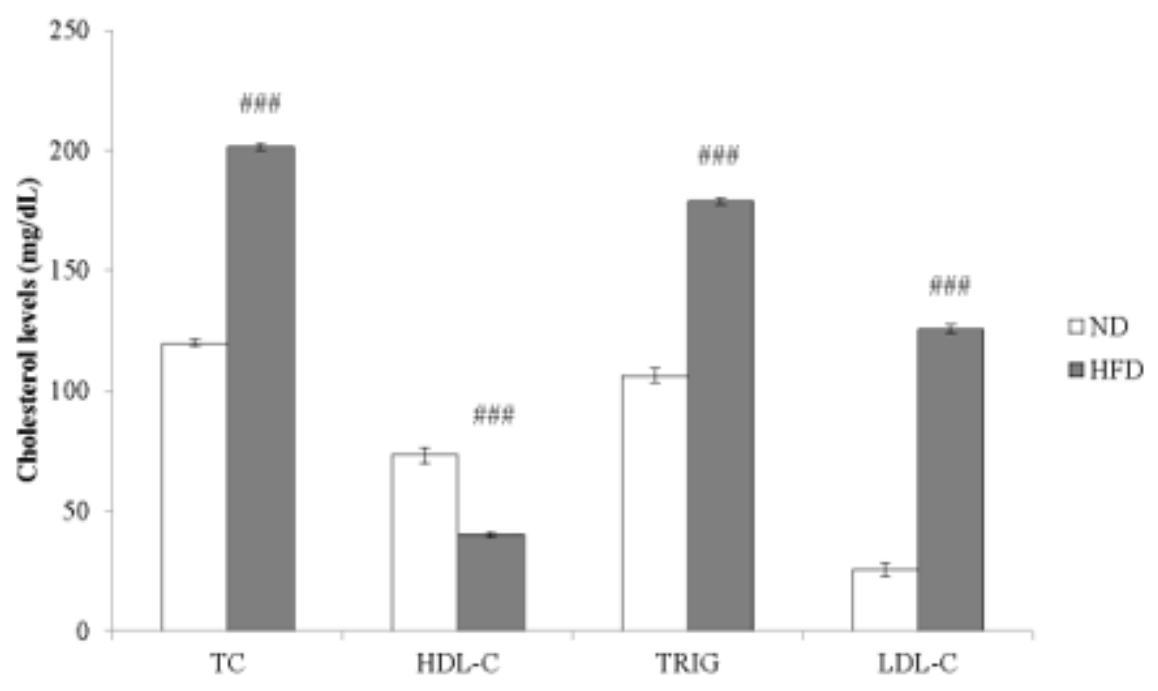

\section{Figure 5}

The effects of the high-fat diet on the plasma lipid levels in the mice. The mice were fed with the normal diet or high-fat diet for 10 weeks. At the end of the feeding, the blood samples were collected from all the animals and used for the lipid profile investigation. The data are expressed as mean \pm SEM $(n=6$ in ND group, $\mathrm{n}=30$ in HFD group). \#\#\#P<0.001 vs. ND group (Student's t-test). Abbreviation: ND, normal diet; HFD, high-fat diet; TC, total cholesterol; HDL-C, high-density lipoprotein cholesterol; TRIG, triglyceride; LDLC, low-density lipoprotein cholesterol.



\section{Figure 6}

The effects of the Chatuphalatika aqueous extract on the plasma total cholesterol levels (A), high-density lipoprotein cholesterol levels (B), triglyceride levels (C) and low-density lipoprotein cholesterol levels (D) in the high-fat diet induced obese mice. The high-fat diet induced obese mice were fed the high-fat diet supplemented with the Chatuphalatika aqueous extract, atorvastatin or distilled water for 6 weeks. At the end of the supplementation, the blood samples were collected from all the animals and used for the lipid profile investigation. The data are expressed as mean \pm SEM ( $n=6$ per group). \#\#\#P<0.001 vs. ND + DDW group (Student's t-test); ${ }^{* \star *} \mathrm{P}<0.001$ vs. HFD + DDW group (one-way ANOVA followed by the StudentNewman-Keuls test). Abbreviations: ND, normal diet; HFD, high-fat diet; DDW, distilled water; ATV, atorvastatin; $\mathrm{CPT}$, Chatuphalatika aqueous extract. 